ITOH, T.

KODAI MATH. SEM. REP

25 (1973), 202-214

\title{
MINIMAL SURFACES IN A RIEMANNIAN MANIFOLD OF CONSTANT CURVATURE
}

\author{
By TAKEHIRo ITOH
}

For surfaces in a 4-dimensional Riemannian manifold of constant curvature, the author [3] proved the following

THEOREM. Let $M$ be a 2-dimensional connected compact Riemannian manifold which is minimally immersed in a unit sphere of dimension 4. If the normal scalar curvature $K_{N}$ is non-zero constant, then $M$ may be regarded as a Veronese surface.

In this paper, he generalizes the above theorem and proves the following

THEOREM. Let $M$ be a 2-dimensional connected compact Riemannian manifold which is minimally immersed in a $(2+\nu)$-dimensional unit sphere $S^{2+\nu}$. If the normal scalar curvature $K_{N}$ is non-zero constant and the square of the second curvature $k_{2}$ is less then $K_{N} / 4$, then $M$ is a generalized Veronese surface.

By a generalized Veronese surface we mean a surface defined by Ōtsuki [6].

\section{$\S 1$. Preliminaries.}

Let $\bar{M}$ be a $(2+\nu)$-dimensional Riemannian manifold of constant curvature $\bar{c}$ and $M$ be a 2-dimensional Riemannian manifold immersed isometrically in $\bar{M}$ by the immersion $x: M \rightarrow \bar{M} . F(\bar{M})$ and $F(M)$ denote the orthonormal frame bundles over $\bar{M}$ and $M$ respectively. Let $B$ be the set of all elements $b=\left(p, e_{1}, e_{2}, e_{3}, \cdots, e_{2+\nu}\right)$ such that $\left(p, e_{1}, e_{2}\right) \in F(M)$ and $\left(p, e_{1}, e_{2}, e_{3}, \cdots, e_{2+\nu}\right) \in F(\bar{M})$ identifying $p \in M$ with $x(p)$ and $e_{i}$ with $d x\left(e_{i}\right), i=1,2$. Then $B$ is naturally considered as a smooth submanifold of $F(\bar{M})$. Let $\bar{\omega}_{A}, \bar{\omega}_{A B}=-\bar{\omega}_{B A}, A, B=1,2,3, \cdots, 2+\nu$, be the basic and connection forms of $\bar{M}$ on $F(\bar{M})$ which satisfy the structure equations:

$$
\begin{aligned}
& d \bar{\omega}_{A}=\sum_{B} \bar{\omega}_{A B} \wedge \bar{\omega}_{B}, \\
& d \bar{\omega}_{A B}=\sum_{C} \bar{\omega}_{A C} \wedge \bar{\omega}_{C B}-\bar{c} \bar{\omega}_{A} \wedge \bar{\omega}_{B} .
\end{aligned}
$$

Recelved May 18, 1972. 
MINIMAL SURFACES IN A RIEMANNIAN MANIFOLD OF CONSTANT CURVATURE 203

In this paper, we use the following convention on the range of indices:

$$
1 \leqq A, B, C, \cdots \leqq 2+\nu, \quad 1 \leqq i, j, \cdots \leqq 2, \quad 3 \leqq \alpha, \beta, \gamma, \cdots \leqq 2+\nu .
$$

Deleting the bars of $\bar{\omega}_{A}, \bar{\omega}_{A B}$ on $B$, as is well known, we have

$$
\begin{aligned}
& \omega_{\alpha}=0, \\
& \omega_{i \alpha}=\sum_{\jmath} A_{\alpha \imath j} \omega_{\jmath}, \quad A_{\alpha \imath \jmath}=A_{\alpha j i}, \\
& d \omega_{i}=\omega_{i \jmath} \wedge \omega_{\jmath}, \quad i \neq j, \\
& d \omega_{i \jmath}=\sum_{k} \omega_{i k} \wedge \omega_{k i}-\Omega_{i \jmath}, \quad \Omega_{\imath \jmath}=\frac{1}{2} \sum_{k, l} R_{\imath j k l} \omega_{k} \wedge \omega_{l}, \\
& R_{\imath j k l}=\bar{c}\left(\delta_{i k} \delta_{j l}-\delta_{i l l} \delta_{j k}\right)+\sum_{\alpha}\left(A_{\alpha i k} A_{\alpha j l}-A_{\alpha i l} A_{\alpha j k}\right), \\
& d \omega_{\alpha \beta}=\sum_{\gamma} \omega_{\alpha \gamma} \wedge \omega_{\gamma \beta}-\Omega_{\alpha \beta}, \quad \Omega_{\alpha \beta}=\frac{1}{2} \sum_{\imath, j} R_{\alpha \beta i j} \omega_{i} \wedge \omega_{\jmath}, \\
& R_{\alpha \beta i \jmath}=\sum_{k}\left(A_{\alpha i k} A_{\beta j k}-A_{\alpha j k} A_{\beta i k}\right) .
\end{aligned}
$$

$M$ is said to be minimal if its mean curvature vector $(1 / 2) \sum_{\alpha, \imath} A_{\alpha i i} e_{\alpha}$ vanishes identically, i.e., if trace $A_{\alpha}=0$ for all $\alpha, A_{\alpha}=\left(A_{\alpha \imath j}\right)$. We say the dimension of the linear space of all second fundamental forms corresponding to normal vectors at $p \in M$ with vanishing trace the minimal index at $p$ and denote it by $m$-index ${ }_{p} M$. We have easily

$$
m \text {-index }{ }_{p} M \leqq 2 \quad \text { at each point } p \in M \text {. }
$$

We denote the square of the norm of the system of all 2nd fundamental forms by

$$
S=\frac{1}{2} \sum_{\alpha \imath, \jmath} A_{\alpha \imath \jmath} A_{\alpha \imath \jmath}=\sum_{\alpha}\left\|A_{\alpha}\right\|^{2}
$$

where for symmetric matrices $A, B$ we define the inner product of $A$ and $B$ by

$$
\langle A, B\rangle=\frac{1}{2} \operatorname{trace} A B \text {. }
$$

We define the normal scalar curvature $K_{N}$ of $M$ in $\bar{M}$ as follows:

$$
K_{N}=\sum_{\imath<j, \alpha<\beta} R_{\alpha \beta i j} R_{\alpha \beta i \jmath}=\sum_{\imath<j, \alpha<\beta}\left(\sum_{k}\left(A_{\alpha i k} A_{\beta j k}-A_{\alpha j k} A_{\beta i k}\right)\right)^{2} .
$$

Now, we assume that $M$ is minimal in $\bar{M}$ and $K_{N}$ is non-zero non $M$. Then we have 


$$
m \text {-index }{ }_{p} M=2 \quad \text { at each point } p \text { on } M \text {. }
$$

Hence, as stated in [5], we can decompose the normal space $N_{p}$ at $p \in M$ as follows:

$$
\begin{aligned}
& N_{p}=N_{p}^{\prime}+O_{p}, \quad N_{p}^{\prime} \perp O_{p}, \quad O_{p}=\phi_{b}^{-1}(0), \\
& \operatorname{dim} N_{p}^{\prime}=2,
\end{aligned}
$$

where $\phi_{b}$ is a linear mapping from $N_{p}$ into the set of all symmetric matrices of order 2 defined by $\phi_{b}\left(\sum_{\alpha} v_{\alpha} e_{\alpha}\right)=\sum_{\alpha} v_{\alpha} A_{\alpha}$. This decomposition does not depend on the choice of a frame $b$,over $p$ and is smooth. Let $B_{0}$ be the set of all $b \in B$ such that $e_{3}, e_{4} \in N_{p}^{\prime}$. Then $B_{0}$ is a smooth submanifold of $B$. On $B_{0}$, we have

$$
\omega_{i \beta}=0 \text {, i.e., } A_{\beta}=0 \text { for } \beta>4 \text {. }
$$

Therefore we have

$$
K_{N}=R_{3412}^{2}=\left(\sum_{k}\left(A_{31 k} A_{42 k}-A_{32 k} A_{41 k}\right)\right)^{2} .
$$

As a special case of [6], we can verify the following

Lemma 1. On $B_{0}$, for a fixed $\beta>4$, we have $\omega_{3 \beta} \equiv \omega_{4 \beta} \equiv 0\left(\bmod \omega_{1}, \omega_{2}\right)$ and $\omega_{3 \beta}=\omega_{4 \beta}=0$ or else $\omega_{3 \beta} \wedge \omega_{4 \beta} \neq 0$.

Now, by virture of Lemma 1 , we can define two linear mappings $\varphi_{11}$ and $\varphi_{12}$ from $M_{p}$ into $O_{p}$ corresponding to the normal vector $e_{3}$ and $e_{4}$ as follows: for any $X \in M_{p}$,

$$
\varphi_{11}(X)=\sum_{\beta>4}\left\|A_{3}\right\| \cdot \omega_{3 \beta}(X) e_{\beta}, \quad \varphi_{12}(X)=\sum_{\beta>4}\left\|A_{4}\right\| \cdot \omega_{4 \beta}(X) e_{\beta}
$$

As stated in [5], these two linear mappings have the same image of the tangent unit sphere $S_{p}^{1}=\left\{X \in M_{p}:\|X\|=1\right\}$ and $\varphi_{11}(X)$ and $\varphi_{12}(X)$ are conjugate to each other with respect to the image when it is an ellipse. We define the second curvature $k_{2}(p)$ of $M$ at $p$ by

$$
k_{2}(p)=\max _{X \in S_{p}^{1}}\left\|\varphi_{11}(X)\right\|=\max _{X \in S_{p}^{1}}\left\|\varphi_{12}(X)\right\| .
$$

It is clear that $k_{2}(p)$ is continuous on $M$.

\section{§ 2. Minimal surfaces with non-zero constant $K_{N}$.}

In this section we assume that $M$ is connected, compact and minimal in $\bar{M}$, $K_{N}$ is non-zero constant and $4 k_{2}^{2}<K_{N}$ at each point of $M$.

LEMMA 2. We have identically

$$
S^{2}=K_{N} \quad \text { on } \quad M
$$


Proof. From (1.10), (1.14) and (1.15), we have

(2.2) $\quad S^{2}-K_{N}=\left\{\left(A_{311}-A_{412}\right)^{2}+\left(A_{312}+A_{411}\right)^{2}\right\}\left\{\left(A_{311}+A_{412}\right)^{2}+\left(A_{312}-A_{411}\right)^{2}\right\} \geqq 0$.

Hence, if it is not identically $S^{2}=K_{N}$, the function $S^{2}-K_{N}$ takes its positive maximum at some point $p_{0} \in M$, because $M$ is compact. Let $U$ be a neighborhood of $p_{0}$ in which we can choose $b \in B_{0}$ such that

$$
A_{3}=\left(\begin{array}{rr}
\lambda & 0 \\
0 & -\lambda
\end{array}\right), \quad A_{4}=\left(\begin{array}{ll}
0 & \mu \\
\mu & 0
\end{array}\right), \quad \lambda^{2}>\mu^{2}>0,
$$

where $\lambda$ and $\mu$ are differentiable functions on $U$. Then we have

$$
K_{N}=4 \lambda^{2} \mu^{2}, \quad S=\lambda^{2}+\mu^{2} .
$$

From (1.1) and (2.3) we have

$$
\begin{aligned}
& d \lambda \wedge \omega_{1}+\left(2 \lambda \omega_{12}-\mu \omega_{34}\right) \wedge \omega_{2}=0, \\
& d \lambda \wedge \omega_{2}-\left(2 \lambda \omega_{12}-\mu \omega_{34}\right) \wedge \omega_{1}=0 \\
& d \mu \wedge \omega_{1}+\left(2 \mu \omega_{12}-\lambda \omega_{34}\right) \wedge \omega_{2}=0, \\
& d \mu \wedge \omega_{2}-\left(2 \mu \omega_{12}-\lambda \omega_{34}\right) \wedge \omega_{1}=0 .
\end{aligned}
$$

Since $K_{N}=4 \lambda^{2} \mu^{2}$ is constant, from (2.4) and (2.5) we have

$$
4 \lambda \mu \omega_{12}=\left(\lambda^{2}+\mu^{2}\right) \omega_{34},
$$

and hence

$$
K_{N} \omega_{12}=\lambda \mu S \omega_{34} .
$$

Differentiating both sides of (2.6), we get

$$
\begin{aligned}
K_{N} d \omega_{12} & =\lambda \mu d S \wedge \omega_{34}+\lambda \mu S d \omega_{34} \\
& =\lambda \mu d S \wedge \omega_{34}-2 \lambda^{2} \mu^{2} S \omega_{1} \wedge \omega_{2}-\lambda \mu S \sum_{\beta>4} \omega_{3 \beta} \wedge \omega_{4 \beta} .
\end{aligned}
$$

On the other hand, since $\omega_{i \beta}=0(\beta>4), \imath=1,2$, we have

$$
d \omega_{i \beta}=\omega_{i 3} \wedge \omega_{3 \beta}+\omega_{i 4} \wedge \omega_{4 \beta}=0,
$$

which reduce to

$$
\begin{aligned}
& \lambda \omega_{3 \beta} \wedge \omega_{1}+\mu \omega_{4 \beta} \wedge \omega_{2}=0, \\
& \lambda \omega_{3 \beta} \wedge \omega_{2}-\mu \omega_{4 \beta} \wedge \omega_{1}=0 .
\end{aligned}
$$

By Cartan's Lemma, we may put 


$$
\begin{aligned}
& \lambda \omega_{3 \beta}=f_{\beta} \omega_{1}+g_{\beta} \omega_{2}, \\
& \mu \omega_{4 \beta}=g_{\beta} \omega_{1}-f_{\beta} \omega_{2},
\end{aligned}
$$

and define two normal vectors $F_{1}=\sum_{4<\alpha} f_{\alpha} e_{\alpha}$ and $G_{1}=\sum_{4<\alpha} g_{\alpha} e_{\alpha}$. Then we have

$$
\lambda \mu \sum_{\beta>4} \omega_{3 \beta} \wedge \omega_{4 \beta}=-\left(\left\|F_{1}\right\|^{2}+\left\|G_{1}\right\|^{2}\right) \omega_{1} \wedge \omega_{2} .
$$

By means of Cartan's Lemma, from (2.4) and (2.5) we have

$$
\begin{aligned}
& 2 \lambda \omega_{12}-\mu \omega_{34}=\lambda_{2} \omega_{1}-\lambda_{1} \omega_{2}, \\
& 2 \mu \omega_{12}-\lambda \omega_{34}=\mu_{2} \omega_{1}-\mu_{1} \omega_{2},
\end{aligned}
$$

putting $d \lambda=\lambda_{1} \omega_{1}+\lambda_{2} \omega_{2}$ and $d \mu=\mu_{1} \omega_{1}+\mu_{2} \omega_{2}$. Thus we get

$$
\left(\lambda^{2}-\mu^{2}\right) \omega_{34}=\left(\lambda_{2} \mu-\lambda \mu_{2}\right) \omega_{1}-\left(\lambda_{1} \mu-\lambda \mu_{1}\right) \omega_{2} .
$$

Since $\lambda \mu=$ constant and hence $\lambda \mu_{i}+\lambda_{i} \mu=0$, we have

$$
\omega_{34}=\frac{2 \mu}{\lambda^{2}-\mu^{2}}\left(\lambda_{2} \omega_{1}-\lambda_{1} \omega_{2}\right)
$$

and

$$
\lambda \mu d S=2 \mu\left(\lambda^{2}-\mu^{2}\right) d \lambda=2 \mu\left(\lambda^{2}-\mu^{2}\right)\left(\lambda_{1} \omega_{1}+\lambda_{2} \omega_{2}\right) .
$$

Hence we have

$$
\lambda \mu d S \wedge \omega_{34}=-4 \mu^{2}\|\nabla \lambda\|^{2} \omega_{1} \wedge \omega_{2},
$$

where $\nabla \lambda$ is the gradient vector of $\lambda$. From (2.7), (2.8) and (2.9), we have

$$
K K_{N}=4 \mu^{2}\|\nabla \lambda\|^{2}+\frac{K_{N} S}{2}-S\left(\left\|F_{1}\right\|^{2}+\left\|G_{1}\right\|^{2}\right),
$$

where $K$ is the Gaussian curvature of $M$. Since $K_{N}>4 k_{2}^{2} \geqq 0,2 k_{2}^{2} \geqq\left\|F_{1}\right\|^{2}+\left\|G_{1}\right\|^{2}$, and $S>0$, we have

$$
K K_{N} \geqq 4 \mu^{2}\|\nabla \lambda\|^{2}+\frac{1}{2} K_{N} S-2 S k_{2}^{2}>0,
$$

so that we get

$$
K>0 \quad \text { on } \quad U \text {. }
$$

From (2.4) and (2.5), we have

$$
\begin{aligned}
& d\left(\lambda^{2}-\mu^{2}\right) \wedge \omega_{1}+4\left(\lambda^{2}-\mu^{2}\right) d \omega_{1}=0, \\
& d\left(\lambda^{2}-\mu^{2}\right) \wedge \omega_{2}+4\left(\lambda^{2}-\mu^{2}\right) d \omega_{2}=0,
\end{aligned}
$$


MINIMAL SURFACES IN A RIEMANNIAN MANIFOLD OF CONSTANT CURVATURE 207

which imply that there exists a neighborhood $V$ of $p_{0}$ where we have isothermal coordinates $(u, v)$ such that

$$
d s^{2}=E\left\{d u^{2}+d v^{2}\right\}, \quad \omega_{1}=\sqrt{ } E d u, \quad \omega_{2}=\sqrt{ } E d v, \quad \sqrt{ } \lambda^{2}-\mu^{2} E=1,
$$

where $E=E(u, v)$ is a positive function on $V$. With respect to these isothermal coordinates, $K$ is given by $K=-(1 / 2 E) \Delta \log E$. Since $\sqrt{\lambda^{2}-\mu^{2}} E=1$ and $\left(\lambda^{2}-\mu^{2}\right)^{2}$ $=S^{2}-K_{N}$, we obtain

$$
K=\frac{\sqrt{\lambda^{2}-\mu^{2}}}{8} \Delta \log \left(S^{2}-K_{N}\right)
$$

which, together with (2.11), implies

$$
\Delta \log \left(S^{2}-K_{N}\right)>0 \quad \text { on } \quad V .
$$

Thus $\log \left(S^{2}-K_{N}\right)$ is a subharmonic function on $V$ and takes its maximum at $p_{0}$ by our assumption, so that $\log \left(S^{2}-K_{N}\right)$ must be constant. Then, (2.12) implies $K=0$, which contradicts $K>0$. Q. E. D.

By Lemma 2, for a frame $b \in B_{0}$ we have $\left\|A_{3}\right\|=\left\|A_{4}\right\|$ and $\left\langle A_{3}, A_{4}\right\rangle=0$. Therefore, on a neighborhood $U(p)$ of $p$ of $M$ we can choose a frame field $b \in B_{0}$ such that

$$
A_{3}=\left(\begin{array}{cc}
k_{1} & 0 \\
0 & -k_{1}
\end{array}\right), \quad A_{4}=\left(\begin{array}{cc}
0 & k_{1} \\
k_{1} & 0
\end{array}\right),
$$

where $k_{1}$ is non-zero constant on $M$. It follows from (2.13) that

$$
\omega_{34}=2 \omega_{12}
$$

and we may put

$$
\begin{aligned}
& k_{1} \omega_{3 \beta}=f_{\beta} \omega_{1}+g_{\beta} \omega_{2}, \\
& k_{1} \omega_{4 \beta}=g_{\beta} \omega_{1}-f_{\beta} \omega_{2}, \quad 4<\beta,
\end{aligned}
$$

as in the proof of Lemma 2. Then, (2.14) implies

$$
\left\|F_{1}\right\|^{2}+\left\|G_{1}\right\|^{2}=2 k_{1}^{2}\left(k_{1}^{2}-K\right),
$$

where $F_{1}=\sum_{4<\beta} f_{\beta} e_{\beta}$ and $G_{1}=\sum_{4<\beta} g_{\beta} e_{\beta}$.

Since $\left\|F_{1}\right\|^{2}+\left\|G_{1}\right\|^{2} \leqq 2 k_{2}^{2}<K_{N} / 2=2 k_{1}^{4}$ and $K=\bar{c}-S=\bar{c}-2 k_{1}^{2}$, we see

$$
K=\text { positive constant on } M \text {. }
$$

Then, we have the following

LEMMA 3. The image of $S_{p}^{1}$ under $\varphi_{11}\left(\right.$ or $\left.\varphi_{12}\right)$ is a circle with constant radius $k_{2}=k_{1} \sqrt{k_{1}^{2}-K}$, where the circle is a point if $k_{2}=0$ on $M$. 
Proof. Putting

$$
l_{2}=\underset{X \in S_{p}^{1}}{\operatorname{Min}}\left\|\varphi_{11}(X)\right\|=\underset{X \in S_{p}^{1}}{\operatorname{Min}}\left\|\varphi_{12}(X)\right\|
$$

we can see

$$
\left(k_{2}^{2}-l_{2}^{2}\right)^{2}=\left(\left\|F_{1}\right\|^{2}-\left\|G_{1}\right\|^{2}\right)^{2}+4\left\langle F_{1}, G_{1}\right\rangle^{2} \geqq 0,
$$

so $\left(k_{2}^{2}-l_{2}^{2}\right)^{2}$ is a differentiable function on $M$, because $\left\{p, F_{1}, G_{1}\right\}$ obey an analogous rule to the rotation of the 2 -frame $\left\{p, e_{3}, e_{4}\right\}$. Hence, if $k_{2}=l_{2}$ does not hold identically on $M$, then $\left(k_{2}^{2}-l_{2}^{2}\right)^{2}$ takes its positive maximum at some point $p_{1}$ on $M$. Let $U_{1}$ be a neighborhood of $p_{1}$ on which $k_{2}>l_{2}$ and we can choose isothermal coordinate $(u, v)$ and a frame $b \in B_{0}$ satisfying (2.13) and

$$
d s^{2}=E\left\{d u^{2}+d v^{2}\right\}, \quad \omega_{1}=\sqrt{E} d u, \quad \omega_{2}=\sqrt{ } E d v
$$

where $E=E(u, v)$ is a positive function on $U_{1}$. Since $\left\|F_{1}\right\|^{2}+\left\|G_{1}\right\|^{2}>0$ on $U_{1}$, we may assume $F_{1} \neq 0$ on a small neighborhood $V_{1}$ of $p_{1}$ in $U_{1}$. Then we can choose a frame field $b \in B_{0}$ satisfying (2.13), (2.16) and

$$
F_{1}=f e_{5}, \quad G_{1}=\sum_{4<\alpha} g_{\alpha} e_{\alpha},
$$

where $f$ is a non-zero differentiable function and $g_{\alpha}$ are differentiable functions on $V_{1}$. Then we have

$$
\begin{array}{ll}
k_{1} \omega_{35}=f \omega_{1}+g_{5} \omega_{2}, & k_{1} \omega_{3 \beta}=g_{\beta} \omega_{2}, \\
k_{1} \omega_{45}=g_{5} \omega_{1}-f \omega_{2}, & k_{1} \omega_{4 \beta}=g_{\beta} \omega_{1}, \quad 5<\beta .
\end{array}
$$

Using these equations and $\omega_{34}=2 \omega_{12}$, from the structure equations we obtain

$$
\begin{aligned}
& d f \wedge \omega_{1}+d g_{5} \wedge \omega_{2}+3 f d \omega_{1}+3 g_{5} d \omega_{2}=\omega_{2} \wedge\left(\sum_{5<\beta} g_{\beta} \omega_{\beta 5}\right), \\
& d g_{5} \wedge \omega_{1}-d f \wedge \omega_{2}+3 g_{5} d \omega_{1}-3 f d \omega_{2}=\omega_{1} \wedge\left(\sum_{5<\beta} g_{\beta} \omega_{\beta 5}\right), \\
& d g_{\beta} \wedge \omega_{2}+3 g_{\beta} d \omega_{2}=-f \omega_{1} \wedge \omega_{\beta 5}-g_{5} \omega_{2} \wedge \omega_{\beta 5}+\omega_{2} \wedge\left(\sum_{\gamma} g_{\gamma} \omega_{\gamma \beta}\right), \\
& d g_{\beta} \wedge \omega_{1}+3 g_{\beta} d \omega_{1}=-g_{5} \omega_{1} \wedge \omega_{\beta 5}+f \omega_{2} \wedge \omega_{\beta 5}+\omega_{1} \wedge\left(\sum_{\gamma} g_{\gamma} \omega_{\gamma \beta}\right),
\end{aligned}
$$

which imply that the complex valued function $E^{3}\left(\left\|G_{1}\right\|^{2}-\left\|F_{1}\right\|^{2}\right)+2 \imath E^{3}\left\langle F_{1}, G_{1}\right\rangle$ is holomorphic in $z=u+i v$, so that

$$
\begin{aligned}
-6 \Delta \log E & =\Delta \log \left\{\left(\left\|F_{1}\right\|^{2}-\left\|G_{1}\right\|^{2}\right)^{2}+4\left\langle F_{1}, G_{1}\right\rangle^{2}\right\} \\
& =\Delta \log \left(k_{2}^{2}-l_{2}^{2}\right)^{2} \quad \text { on } V_{1} .
\end{aligned}
$$

Since $K$ is given by $K=-(1 / 2 E) \Delta \log E$ and is positive from (2.15), (2.17) implies that $\log \left(k_{2}^{2}-l_{2}^{2}\right)^{2}$ is a subharmonic function on $V_{1}$. Since $\left(k_{2}^{2}-l_{2}^{2}\right)^{2}$ takes its positive 
maximum at $p_{1}$ in $V_{1}, \log \left(k_{2}^{2}-l_{2}^{2}\right)^{2}$ must be constant so that (2.7) implies $K=0$, which contradicts $K>0$. Thus, $k_{2}=l_{2}$ holds at every point on $M$. Furthermore, we have $\left\|F_{1}\right\|=\left\|G_{1}\right\|$ and $\left\langle F_{1}, G_{1}\right\rangle=0$ for any frame $b \in B_{0}$ satisfying (2.13). Since $2 k_{2}^{2}=\left\|F_{1}\right\|^{2}+\left\|G_{1}\right\|^{2}=2 k_{1}^{2}\left(k_{1}^{2}-K\right)$ is constant on $M, k_{2}=l_{2}=k_{1} \sqrt{k_{1}^{2}-\bar{K}}$ is constant on $M$. Q. E.D.

By Lemma 3, if $k_{2}=0$ on $M$, then the geodesic codimension of $M$ in $\bar{M}$ is 2, so that $M$ is a Veronese surface (see [3]). If $k_{2} \neq 0$ on $M$, then by Lemma 2 and 3 , on a neighborhood $U(p)$ of a $p$ of $M$ we can choose a frame field $b \in B_{0}$ satisfying (2.13) and

$$
\begin{aligned}
& k_{1} \omega_{35}=k_{2} \omega_{1}=k_{1} \omega_{46} \\
& k_{1} \omega_{36}=k_{2} \omega_{2}=-k_{1} \omega_{45}, \quad \omega_{3 \beta}=\omega_{4 \beta}=0, \quad 6<\beta,
\end{aligned}
$$

where $k_{2}$ is non-zero constant on $M$. Then, from (2.14) and (2.18) we obtain

$$
\omega_{56}=3 \omega_{12}
$$

and we may put

$$
\begin{aligned}
& k_{2} \omega_{5 \beta}=f_{\beta} \omega_{1}+g_{\beta} \omega_{2} \\
& k_{2} \omega_{6 \beta}=g_{\beta} \omega_{1}-f_{\beta} \omega_{2}, \quad 6<\beta,
\end{aligned}
$$

where $f_{\beta}$ and $g_{\beta}$ are differentiable functions on $U(p)$. We consider two linear mappings $\varphi_{21}$ and $\varphi_{22}$ from $M_{p}$ into $N_{p}$ as follows

$$
\begin{aligned}
& \varphi_{21}(X)=\sum_{\beta} k_{2} \omega_{5 \beta}(X) e_{\beta}=\omega_{1}(X) F_{2}+\omega_{2}(X) G_{2}, \\
& \varphi_{22}(X)=\sum_{\beta} k_{2} \omega_{6 \beta}(X) e_{\beta}=\omega_{1}(X) G_{2}-\omega_{2}(X) F_{2},
\end{aligned}
$$

where $X$ is a tangent vector to $M$ and $F_{2}=\sum_{6<\beta} f_{\beta} e_{\beta}$ and $G_{2}=\sum_{6<\beta} g_{\beta} e_{\beta}$ are normal vector fields on $U(p)$. Using (2.19) and the structure equations, we obtain

$$
\left\|F_{2}\right\|^{2}+\left\|G_{2}\right\|^{2}=k_{2}^{2}\left(\frac{2 k_{2}^{2}}{k_{1}^{2}}-3 K\right)=\text { constant on } M \text {. }
$$

In the same manner as the proof of Lemma 3, we can prove the following

LEMMA 4. If $k_{2}=$ constant $\neq 0$ on $M$, the image of $S_{p}^{1}$ under $\varphi_{21}$ (or $\left.\varphi_{22}\right)$ is a circle with constant radius $k_{2} \sqrt{k_{2}^{2} / k_{1}^{2}-3 \bar{K} / 2}$, where the circle is a point if $2 k_{2}^{2}=3 k_{1}^{2} K$ on $M$.

If $2 k_{2}^{2}=3 k_{1}^{2} K$ on $M$, then the geodesic codimension of $M$ is 4 , because $\omega_{i_{\beta}}=0$ $(4<\beta), \omega_{3 \gamma_{1}}=\omega_{4 \gamma_{1}}=0\left(6<\gamma_{1}\right)$ and $\omega_{5 \gamma_{2}}=\omega_{6 \gamma_{2}}=0\left(8<\gamma_{2}\right)$. Henceforth, we may consider the case $2 k_{2}^{2} \neq 3 k_{1}^{2} K$ on $M$. Then, by Lemmas 2,3 and 4 , on a neighborhood of a point $p$ on $M$ we can choose a frame field $b \in B_{0}$ satisfying (2.13), (2.18) and the following conditions: 


$$
\begin{aligned}
& k_{2} \omega_{57}=k_{3} \omega_{1}=k_{2} \omega_{68}, \\
& k_{2} \omega_{58}=k_{3} \omega_{2}=-k_{2} \omega_{67}, \quad \omega_{5 \gamma}=\omega_{6 \gamma}=0, \quad 8<\gamma,
\end{aligned}
$$

where $k_{3}$ is a non-zero constant on $M$. From the above equations we get

$$
\omega_{78}=4 \omega_{12} .
$$

We use the following convention about indices:

$$
I_{0}=\{1,2\}, \quad I_{t}=\{2 t+1,2 t+2\}, \quad t=1,2, \cdots, m,
$$

and if we write $\alpha_{1}, \alpha_{2} \in I_{t}$, then $\alpha_{1}<\alpha_{2}$.

Now we shall prove the following

THEOREM 1. Let $M$ be a 2-dimensional connected compact Riemannan manifold which is isometrically and minimally immersed in a Riemannian manfold $\bar{M}$ of constant curvature $\bar{c}$. If the normal scalar curvature $K_{N}$ is non-zero constant on $M$ and the square of the second curvature $k_{2}$ is less than $K_{N} / 4$, then the geodesic codimension of $M$ in $\bar{M}$ is even $2 m$ ( $m$ is a positive integer), and we can choose a frame $b \in B_{0}$ such that

$$
\begin{aligned}
& k_{t-1} \omega_{\alpha_{1} \beta_{1}}=k_{t} \omega_{1}=k_{t-1} \omega_{\alpha_{2} \beta_{2}}, \quad \omega_{\alpha_{1} \gamma}=0, \\
& k_{t-1} \omega_{\alpha_{1} \beta_{2}}=k_{t} \omega_{2}=-k_{t-1} \omega_{\alpha_{2} \beta_{1}}, \quad \omega_{\alpha_{2} \gamma}=0 \text {, } \\
& \alpha_{1}, \alpha_{2} \in I_{t-1}, \quad \beta_{1}, \beta_{2} \in I_{t}, \quad 2 t+2<\gamma, \\
& t=1,2, \cdots, m,
\end{aligned}
$$

where $k_{0}=1$ and $k_{t}(2 \leqq t \leqq m)$ are non-zero constant on $M$. Furthermore, we obtain

$$
\begin{gathered}
\omega_{\alpha_{1} \alpha_{2}}=(t+1) \omega_{12}, \quad \alpha_{1}, \alpha_{2} \in I_{t} \quad(t=1,2, \cdots, m), \\
(t+1) K=\frac{2 k_{t}^{2}}{k_{t-1}^{2}}-\frac{2 k_{t+1}^{2}}{k_{t}^{2}} \quad(t=1, \cdots, m-1), \\
(m+1) K=\frac{2 k_{m}^{2}}{k_{m-1}^{2}} .
\end{gathered}
$$

Proof. By induction with respect to $t$, we shall prove the theorem. For $t=1$, 2 and 3, we proved our assertions by Lemmas 2, 3 and 4 respectively. Hence, we suppose that our (2.21), (2.22) and (2.23) hold for all $t \leqq t_{0}$. In this case, we shall prove that our assertion holds for $t_{0}+1$. Then, since $\omega_{\alpha_{1} \gamma}=\omega_{\alpha_{2} \gamma}=0, \alpha_{1}, \alpha_{2} \in I_{t_{0}-1}$, $2 t_{0}+2<\gamma$, we have

$$
\begin{aligned}
& k_{t_{0}} \omega_{\beta_{1} \gamma} \wedge \omega_{1}+k_{t_{0}} \omega_{\beta_{2} r} \wedge \omega_{2}=0, \\
& k_{t_{0}} \omega_{\beta_{1} \gamma} \wedge \omega_{2}-k_{t_{0}} \omega_{\beta_{2} r} \wedge \omega_{1}=0, \quad \beta_{1}, \beta_{2} \in I_{t_{0}},
\end{aligned}
$$

which, together with Cartan's lemma, imply that we may put 


$$
\begin{aligned}
& k_{t_{0}} \omega_{\beta_{1 \gamma}}=f_{\gamma} \omega_{1}+g_{\gamma} \omega_{2}, \\
& k_{t_{0}} \omega_{\beta_{2 \gamma}}=g_{\gamma} \omega_{1}-f_{\gamma} \omega_{2}, \quad 2 t_{0}+2<\gamma,
\end{aligned}
$$

and define two normal vector fields $F_{t_{0}}=\sum_{\gamma} f_{\gamma} e_{\gamma}$ and $G_{t_{0}}=\sum_{\gamma} g_{\gamma} e_{\gamma}$. We consider two linear mappings $\varphi_{t_{0} 1}$ and $\varphi_{t_{0}{ }^{2}}$ from $M_{p}$ into $N_{p}$ as follows:

$$
\begin{aligned}
& \varphi_{t_{0} 1}(X)=\sum_{\gamma} k_{t_{0}} \omega_{\beta_{1} \gamma}(X) e_{\gamma}=\omega_{1}(X) F_{t_{0}}+\omega_{2}(X) G_{t_{0}}, \\
& \varphi_{t_{0} 2}(X)=\sum_{\gamma} k_{t_{0}} \omega_{\beta_{2} \gamma}(X) e_{\gamma}=\omega_{1}(X) G_{t_{0}}-\omega_{2}(X) F_{t_{0}},
\end{aligned}
$$

where $X$ is a tangent vector to $M$. Putting

$$
k_{t_{0}+1}=\underset{X \in S_{p}^{1}}{\operatorname{Max}}\left\|\varphi_{t_{0} 1}(X)\right\|=\underset{X \in S_{p}^{1}}{\operatorname{Max}}\left\|\varphi_{t_{0} 2}(X)\right\| \quad \text { and } \quad t_{t_{0}+1}=\underset{X \in S_{p}^{1}}{\operatorname{Min}}\left\|\varphi_{t_{0} 1}(X)\right\|=\underset{X \in S_{p}^{1}}{\operatorname{Min}}\left\|\varphi_{t_{0} 2}(X)\right\|
$$

we can see

$$
\left(k_{t_{0}+1}^{2}-l_{t_{0}+1}^{2}\right)^{2}=\left(\left\|F_{t_{0}}\right\|^{2}-\left\|G_{t_{0}}\right\|^{2}\right)^{2}+4\left\langle F_{t_{0}}, G_{t_{0}}\right\rangle^{2},
$$

so $\left(k_{t_{0}+1}^{2}-l_{t_{0}+1}^{2}\right)^{2}$ is a differentiable function on $M$, because $\left\{p, F_{t_{0}}, G_{t_{0}}\right\}$ obey an analogous rule to the rotation of the 2 -frame $\left\{p, e_{\beta_{1}}, e_{\beta_{2}}\right\}$. Hence, similarly to the proof of Lemma 3 , we can see that $k_{t_{0}+1}=l_{t_{0}+1}$ holds everywhere on $M$. On the other hand, since $\omega_{\beta_{1} \beta_{2}}=\left(t_{0}+1\right) \omega_{12}$, we get

$$
2 k_{t_{0}+1}^{2}=\left\|F_{t_{0}}\right\|^{2}+\left\|G_{t_{0}}\right\|^{2}=k_{t_{0}}^{2}\left(\frac{2 k_{t_{0}}^{2}}{k_{t_{0}-1}^{2}}-\left(t_{0}+1\right) K\right)=\text { constant on } M \text {. }
$$

If $2 k_{t_{0}}^{2}=(t+1) k_{t_{0}-1}^{2} K$ on $M$, we can see that the geodesic codimension of $M$ is $2 t_{0}$ and (2.24) holds. Therefore, we consider the case $2 k_{t_{0}}^{2} \neq\left(t_{0}+1\right) k_{t_{0}-1}^{2} K$ on $M$. Then, by the above argument, we can choose a frame field $b \in B_{0}$ satisfying (2.21) for all $t \leqq t_{0}$ and

$$
\begin{array}{ll}
k_{t_{0}} \omega_{\hat{\beta}_{1} \gamma_{1}}=k_{t_{0}+1} \omega_{1}=k_{t_{0}} \omega_{\beta_{2} \gamma_{2}}, & \omega_{\beta_{1 \gamma}}=0, \\
k_{t_{0}} \omega_{\beta_{1} \gamma_{2}}=k_{t_{0}+1} \omega_{2}=-k_{t_{0}} \omega_{\beta_{2} \gamma_{1}}, & \omega_{\beta_{2 \gamma}}=0, \\
\gamma_{1}, \gamma_{2} \in I_{t_{0}+1}, & 2 t_{0}+4<\gamma,
\end{array}
$$

where $k_{t_{0}+1}$ is non-zero constant on $M$, which imply that (2.21), (2.22) and (2.23) hold for $t_{0}+1$. Thus, it is clear that the geodesic codimension of $M$ in $\bar{M}$ is even $2 m$. Then, since we have $\omega_{2 m+1}{ }_{2 m+2}=(m+1) \omega_{12}$ and $\omega_{2 m+1}=\omega_{2 m_{r}}=0(2 m+2<\gamma)$, we obtain (2.24). Q.E.D.

\section{$\S 3$. The proof of the main theorem.}

By an analogous computation to the one in $\S 4$ in [7], from Theorem 1 we obtain the following 
THEOREM 2. Let $M$ be a 2-dimensional connected compact Riemannian manifold which is isometrically and minimally immersed in a Riemannian manifold $\bar{M}$ of constant curvature $\bar{c}$. If the normal scalar curvature $K_{N}$ is non-zero constant on $M$ and if the square of the second curvature $k_{2}$ is less than $K_{N} / 4$, then the geodesic codimension is even $2 m$ and the Gaussian curvature $K$ is positive constant, and supposing $K=1$, there exist $m$ constants $b_{t}=(m-t+1)(m+t+2) / 41 \leqq t \leqq m$, and $m$ complex normal vector fields $\xi_{1}, \cdots, \xi_{m}$ such that

$$
\begin{aligned}
& \xi_{t} \cdot \xi_{s}=\xi_{t} \cdot \bar{\xi}_{s}=0, \quad t \neq s, \\
& \xi_{t} \cdot \xi_{t}=0, \quad \xi_{t} \cdot \bar{\xi}_{t}=2, \quad t=2,3, \cdots, m
\end{aligned}
$$

and

$$
\begin{aligned}
d x= & \frac{1}{h}\left(\bar{\xi}_{0} d z+\xi_{0} d \bar{z}\right), \quad \xi_{0}=e_{1}+\imath e_{2}, \\
\bar{D} \xi_{0}= & \frac{1}{h} \xi_{0}(\bar{z} d z-z d \bar{z})+\frac{2 \sqrt{b_{1}}}{h} \xi_{1} d \bar{z}, \quad h=1+z \bar{z}, \\
\bar{D} \xi_{1}= & -\frac{2 \sqrt{b_{1}}}{h} \xi_{0} d z+\frac{2}{h} \xi_{1}(\bar{z} d z-z d \bar{z})+\frac{2 \sqrt{b_{2}}}{h} \xi_{2} d \bar{z}, \\
& \ldots \ldots \ldots \cdots, \\
\bar{D} \xi_{t}= & -\frac{2 \sqrt{b_{t}}}{h} \xi_{t-1} d z+\frac{t+1}{h} \xi_{\iota}(\bar{z} d z-z d \bar{z})+\frac{2 \sqrt{b_{t+1}}}{h} \xi_{t+1} d \bar{z}, \\
& \ldots \ldots \cdots \cdots \cdots, \\
\bar{D} \xi_{m}= & -\frac{2 \sqrt{b_{m}}}{h} \xi_{m-1} d z+\frac{m+1}{h} \xi_{m}(\bar{z} d z-z d \bar{z}),
\end{aligned}
$$

where $z$ is an isothermal complex coordinate of $M$ and $\bar{D}$ denotes the covarnant differentiation of $\bar{M}$.

In Theorem 2 we may consider $\bar{M}^{2+2 m}=\bar{M}=S^{2-2 m}(R)$, where $S^{2+2 m}(R)$ denotes the $(2+2 m)$-sphere of radius $R$ :

$$
\frac{1}{R^{2}}=\bar{c}=\frac{(m+1)(m+2)}{2} .
$$

We regard as $S^{2+2 m}(R) \subset E^{3+2 m}$ and put

$$
\frac{x}{R}=e_{3+2 m} .
$$

By (3.1) we have

$$
d x=R e_{3-2 m}=\frac{1}{h}\left(\bar{\xi}_{0} d z+\xi_{0} d \bar{z}\right) .
$$

From (II) in Theorem 2 and the above relation, we have easily 


$$
\begin{aligned}
d \xi_{0}= & \frac{1}{h} \xi_{0}(\bar{z} d z-z d \bar{z})+\frac{2 \sqrt{b_{1}}}{h} \xi_{1} d z-\frac{2}{R h} e_{3+2 m} d z \\
d \xi_{1}= & \frac{2 \sqrt{b_{1}}}{h} \xi_{0} d z+\frac{2}{h} \xi_{1}(\bar{z} d z-z d \bar{z})+\frac{2 \sqrt{b_{2}}}{h} \xi_{z} d \bar{z} \\
& \cdots \cdots \cdots \cdots \cdots \\
d \xi_{t}= & -\frac{2 \sqrt{b_{t}}}{h} \xi_{t-1} d z+\frac{t+1}{h} \xi_{t}(\bar{z} d z-z d \bar{z})+\frac{2 \sqrt{b_{t+1}}}{h} \xi_{t+1} d \bar{z} \\
& \cdots \cdots \cdots \cdots \cdots \\
d \xi_{m}= & -\frac{2 \sqrt{b_{m}}}{h} \xi_{m-1} d z+\frac{m+1}{h} \xi_{m}(\bar{z} d z-z d \bar{z})
\end{aligned}
$$

where $d$ denotes the ordinary differential operator in $E^{3+2 m}$. Since equations (3.3) are the same ones as (II) in Theorem 2 in Ōtsuki [7] when we put formally $P=-(1 / R) e_{3+2 m}$ in the case $\bar{M}^{n+2 m}=E^{n+2 m}, M$ is congruent to the surface given by

$$
\begin{aligned}
x= & \frac{\sqrt{m !}}{(m+2) \sqrt{(2 m+2)(2 m+1) \cdots(m+3)(1+z \bar{z})^{m+1}}} \\
\times & {\left[\sum_{\jmath=0}^{m}(-1)^{\jmath+1}\left\{\sum_{s=0}^{j}(-1)^{s}\left(\begin{array}{c}
2 m+2-j \\
m+1-s
\end{array}\right)\left(\begin{array}{l}
\jmath \\
s
\end{array}\right)(z \bar{z})^{s}\right\}\left(\bar{z}^{m+1-\jmath} A_{j}+z^{m+1-\jmath} \bar{A}_{\jmath}\right)\right.} \\
& \left.+(-1)^{m} \sum_{s=0}^{m+1}(-1)^{s}\left(\begin{array}{c}
m+1 \\
s
\end{array}\right)^{2}(z \bar{z})^{s} A_{m+1}\right]
\end{aligned}
$$

where $A_{0}, A_{1}, \cdots, A_{m+1}$ are constant complex vectors in $C^{m+2}$ such that

$$
\begin{aligned}
& A_{t} \cdot A_{t}=0, \quad t=0,1, \cdots, m, \quad A_{m+1}=\bar{A}_{m+1}, \\
& A_{t} \cdot A_{s}=A_{t} \cdot \bar{A}_{s}=0, \quad t \neq s, \quad t, s=0,1, \cdots, m+1, \\
& A_{0} \cdot \bar{A}_{0}=2, \quad A_{t} \cdot \bar{A}_{t}=2\left(\begin{array}{c}
2 m+2 \\
t
\end{array}\right), \quad t=1,2, \cdots, m+1 .
\end{aligned}
$$

Thus we have proved that $M$ may be regarded as a generalized Veronese surface of index $m$ defined by Ōtsuki [7].

\section{REFERENCES}

[1] Chern, S. S., M. Do Carmo, and S. Kobayashi, Minimal submanifolds of a sphere with second fundamental form of constant length. Functional analysis and related fields, Proc. Conf. in Honor of Marshall Stone, Springer, Berlin (1970).

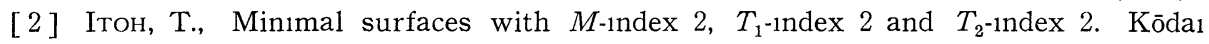
Math. Sem. Rep. 24 (1972), 1-16.

[3] — Minimal surfaces in 4-dimensional Riemannian manifolds of constant curvature. Kōdaı Math. Sem. Rep. 24 (1972), 451-458. 
[4] Little, J. A., On singularities of submanifolds of higher dimensional Euclidean spaces. Ann. Mat. Pura Appl. 83 (1969), 261-336.

[5] Ōtsukı, T., A Theory of Riemannıan submanıfolds. Kōdaı Math. Sem. Rep. 20 (1968), 282-295.

[6] —, Minımal submanıfolds with $M$-ındex 2. J. Diff. Geometry 6 (1971), 193-211.

[7] Minımal submanıfolds with $M$-1ndex 2 and generalized Veronese surfaces. J. Math. Soc. Japan 24 (1972), 89-122.

Department of the Foundations of Mathematical Sciences, TOKyo University of Education. 